\title{
Symptomatic changes in postmenopause with different methods of hormonal therapy
}

\author{
Marcelino Hernández-Valencia*, Nydia Cordova, Antonio Vargas, Lourdes Basurto, \\ Renata Saucedo, Carlos Vargas, Miriam Ruiz, Leticia Manuel-Apolinar, Arturo Zárate
}

Endocrine Research Unit, Specialties Hospital, National Medical Center, Instituto Mexicano del Seguro Social, México City, Mexico; *Corresponding author: mhernandezvalencia@prodigy.net.mx

Received 20 April 2011; revised 30 May 2011; accepted 20 July 2011.

\begin{abstract}
Objective: the diversity of opinions on the adverse effects of medications used to treat postmenopausal symptoms has prompted the use of various routes and mechanisms of action that need to be explored because bioavailability of the medications can vary. In order to select the appropriate route of administration for hormonal therapy $(\mathrm{HT})$, it is necessary to determine baseline therapeutic efficacy. Design: we designed a prospective, randomized study consisting of four groups of postmenopausal women: group 1 received oral conjugated estrogens, group 2 received a synthethic steroid, group 3 received estradiol nasally in spray form, and group 4 used transdermal estradiol in the form of patches. Criteria used to evaluate effectiveness was the Greene scale, which evaluate six components. These criteria were applied to each patient before hormonal intervention and then each month for 6 months. Luteinizing hormone (LH), follicle stimulating horone (FSH) and estradiol concentration were determined by chemiluminescence. Student's t-test was used for intra-group comparisons before and after treatment. Results: There was a significant decrease in the vasomotor and sexual component $(p<0.05)$ with the use of four HT types. For depression, a difference was observed with synthetic steroids and oral estrogens. Upon analyzing the somatic component there was a decrease in symptoms with nasal and transdermal routes. Psychological changes were observed with the use of oral synthethic steroids and transdermal patches. Anxiety component demonstrated differences with nasal spray and oral estrogens, although all HT forms in this component showed a pattern of irregular changes.
\end{abstract}

Conclusions: changes in the response could be due each route of administration and medication used. Absorption variability may exist, which has repercussions in the control of symptoms and should be taken into consideration when selecting the appropriate route of administration for patients beginning HT.

Keywords: Hormonal Therapy; Transdermal Estradiol; Tibolone; Oral Estrogen; Nasal Estradiol; Postmenopause

\section{INTRODUCTION}

Postmenopause is the stage of life when a profound decrease occurs in the circulating concentration of estrogens, inducing the appearance of characteristic psychosomatic symptoms [1,2]. Absence of this hormone is the reason for estrogens receptors not exercising intracellular messages in different tissues sensitive to estrogens, producing a functional derangement clinically translated by menopause symptoms that compromise quality of life $[3,4]$.

Due to a variety of opinions on the adverse effects of medications used to treat menopause [5,6], diverse routes and mechanisms of action have been used, which need to be explored as to their responses. Differences in bioavailability of medications can vary when passing through the intestine and liver $[7,8]$. The actual tendency is to find the baseline therapeutic efficacy when choosing the appropriate route of hormonal therapy (HT) administration to provide relief for the predominant symptoms that each patient presents at the first medical visit, since both doctors and women are seeking the optimal way to use HT [9,10].

This study was undertaken to compare the effects of different HT forms and routes of administration used to improve each of the symptomatic components in postmenopause. 


\section{MATERIALS AND METHODS}

This was a prospective, longitudinal and descriptive study that included 160 healthy postmenopausal women who were recruited from Department of Health Promotion. Subjects were evaluated for admission into the study in the Endocrine Research Unit of the Specialties Hospital, National Medical Center, Instituto Mexicano del Seguro Social. According to patient selection, four study groups were formed. Each group was comprised of 40 patients randomly assigned. Group 1 received oral conjugated estrogens (Premarin $0.625 \mathrm{mg}$ ). Group 2 received a synthetic steroid (Tibolone $2.5 \mathrm{mg}$ ). Group 3 received estradiol nasally in the form of a spray in each nostril (Armistor $300 \mu \mathrm{g}$ ). Group 4 used transdermal estradiol in the form of patches that were changed every 4 days (Estraderm $1.5 \mathrm{mg}$ ). All groups received a daily dose at night and patches were also changed during the night, administered for a period of 6 months.

Included patients were postmenopasual, confirmed hormonally by estradiol concentrations $<10 \mathrm{pg} / \mathrm{mL}$ and FSH $>40 \mathrm{mIU} / \mathrm{mL}$. Uterine ultrasound demonstrated normal endometrium, and cervical cytology and mammography were without changes. As a study criterion, subjects had no history of associated treatments to correct psychoaffective symptomatology characteristic of postmenopause. Exclusion criteria were nasal diseases, allergies, history of abnormal bleeding and thrombophlebitis.

In none of the patients was a progestin associated with HT due to the short length of the study where our primary objective was to observe affective changes on postmenopausal symptoms.

All patients gave informed consent upon admission to the study, and the study was approved by the Ethics Committee from our hospital.

\subsection{Clinical Evaluation}

A complete clinical evaluation was performed before beginning treatment and then monthly until 6 months of follow-up, where at each medical visit the response on the climacteric symptomatology was also evaluated, as well as occurrence of undesirable effects, and blood samples were obtained for hormonal determinations.

The Greene scale [11] was the criteria used for evaluation of symptoms and included six components: vasomotor, sexual, depression, somatic, psychological and anxiety. This scale was applied to each patient before hormonal intervention and monthly during follow-up. This scale indicates a weighted global score that quantifies the incidence and intensity of postmenopausal symptoms.

\subsection{Hormonal Determinations}

Serum from each subject was kept in aliquots (1 - 3 $\mathrm{mL}$ ), and hormonal determinations were tested using commercial kits in accordance with established protocols. Concentrations of luteinizing hormone (LH), follicle stimulanting hormone (FSH) and estradiol were determined by chemiluminescence (Immulite, Diagnostic Products Corporation, Los Angeles, CA) with a sensitivity of $0.1 \mathrm{mIU} / \mathrm{mL}$ for FSH, $0.1 \mathrm{mIU} / \mathrm{mL}$ for $\mathrm{LH}$ and 7.0 $\mathrm{pg} / \mathrm{mL}$ for estradiol. All determinations had a coefficient of variation intra- and inter-assay of $6.7 \%$ and $3.7 \%$, respectively.

\subsection{Statistical Analysis}

Description of the results was performed using dispersion measures and shown as mean \pm standard deviation $(\mathrm{M} \pm \mathrm{SD})$. Comparison between groups was determined by two-way analysis of variance (ANOVA), with a relevant post hoc test. Differences were considered statistically significant if $p<0.05$.

\section{RESULTS}

Clinical characteristics showed no statistically significant differences among the study groups (Table 1). All patients included in this study completed 6 months of treatment, and in no cases were there intense adverse symptoms warranting HT suspension.

Hormonal determinations demonstrated good pharmacological response because the baseline concentrations of gonadotrophins confirmed a significant statistical decrease $(p<0.05)$, taking into consideration all patients included in the study with LH $32.6 \pm 4.2$ to $12.2 \pm$ $3.1 \mathrm{mIU} / \mathrm{mL}$ and FSH $81.6 \pm 9.4$ to $26.4 \pm 6.3 \mathrm{mIU} / \mathrm{mL}$, as well as an increase in estradiol of $10.8 \pm 2.4$ to $94.7 \pm$ $8.6 \mathrm{pg} / \mathrm{mL}$ after 3 months and at the end of HT.

Greene scale score showed changes in symptomatology from the first month of HT in the four groups, but without significant differences. Statistical differences in the study were observed at 3 months for some of the scale components, but 6 months of HT for each scale component was considered for analysis.

Table 1. Clinical characteristics of the four study groups.

\begin{tabular}{cccccc}
\hline Variable & Nasal & Synthetic & Conjugated Transdermal & $p$ \\
\hline Age & $49.3 \pm 4.2$ & $47.9 \pm 2.7$ & $49.6 \pm 3.6$ & $48.1 \pm 2.2$ & NS \\
BMI & $24.6 \pm 2.8$ & $25.4 \pm 1.4$ & $25.1 \pm 1.6$ & $24.9 \pm 2.1$ & NS \\
$\begin{array}{c}\text { Age at meno- } \\
\text { pause }\end{array}$ & $49.3 \pm 2.5$ & $46.8 \pm 0.5$ & $48.7 \pm 6.2$ & $47.6 \pm 1.2$ & $\mathrm{NS}$ \\
$\begin{array}{c}\text { Months of } \\
\text { menopause } \\
\begin{array}{c}\text { Total Greene } \\
\text { score }\end{array}\end{array}$ & $14.2 \pm 1.2$ & $14.1 \pm 1.1$ & $14.8 \pm 2.6$ & $16.2 \pm 1.3$ & $\mathrm{NS}$ \\
\hline
\end{tabular}

BMI, body mass index; NS, not significant. 
Vasomotor component demonstrated a significant decrease $(p<0.05)$ with the use of the four types of HT, maintaining the pattern of decrease in this symptom observed at 3 months (Figure 1(A)). There was a decrease in the sexual component with the same use of HT, although with an irregular pattern of decrease (Figure 1(B)).

For the depressive component, a difference was observed with the use of oral synthetic steroids and conjugated oral estrogens, with a pattern of constant decrease of symptoms even with medications where there was no significant difference (Figure 2(A)). On analyzing the somatic component, a decrease in symptoms was observed with the use of the nasal spray and transdermal patches, where a constant and profound decrease was found as opposed to the other forms of therapy where irregular changes existed (Figure 2(B)).
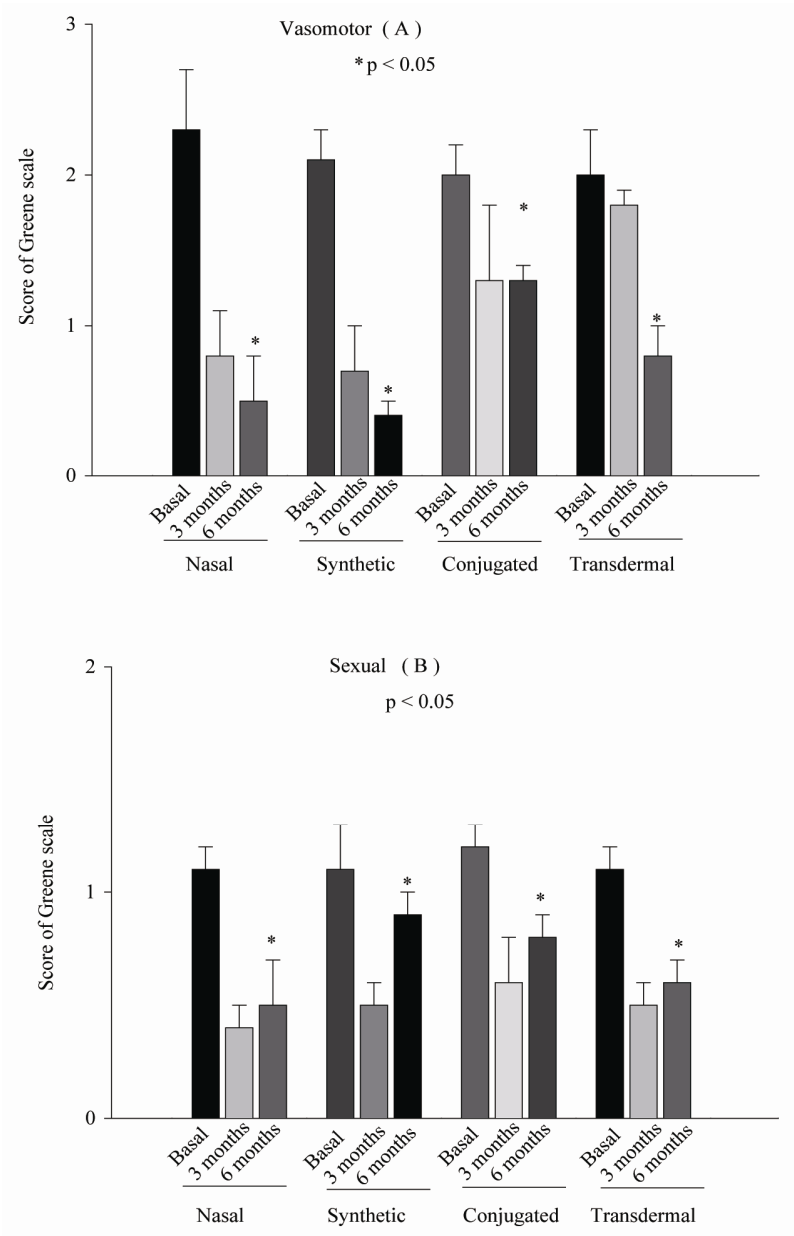

Figure 1. Two components of the Greene scale are observed where the vasomotor component (Figure 1(A)) demonstrates a statistically significant decrease $(p<0.05)$ with the use of all types of hormonal therapy. The sexual component (Figure 1(B)) demonstrates same statistical differences in all therapies, in addition to an irregular score distribution.
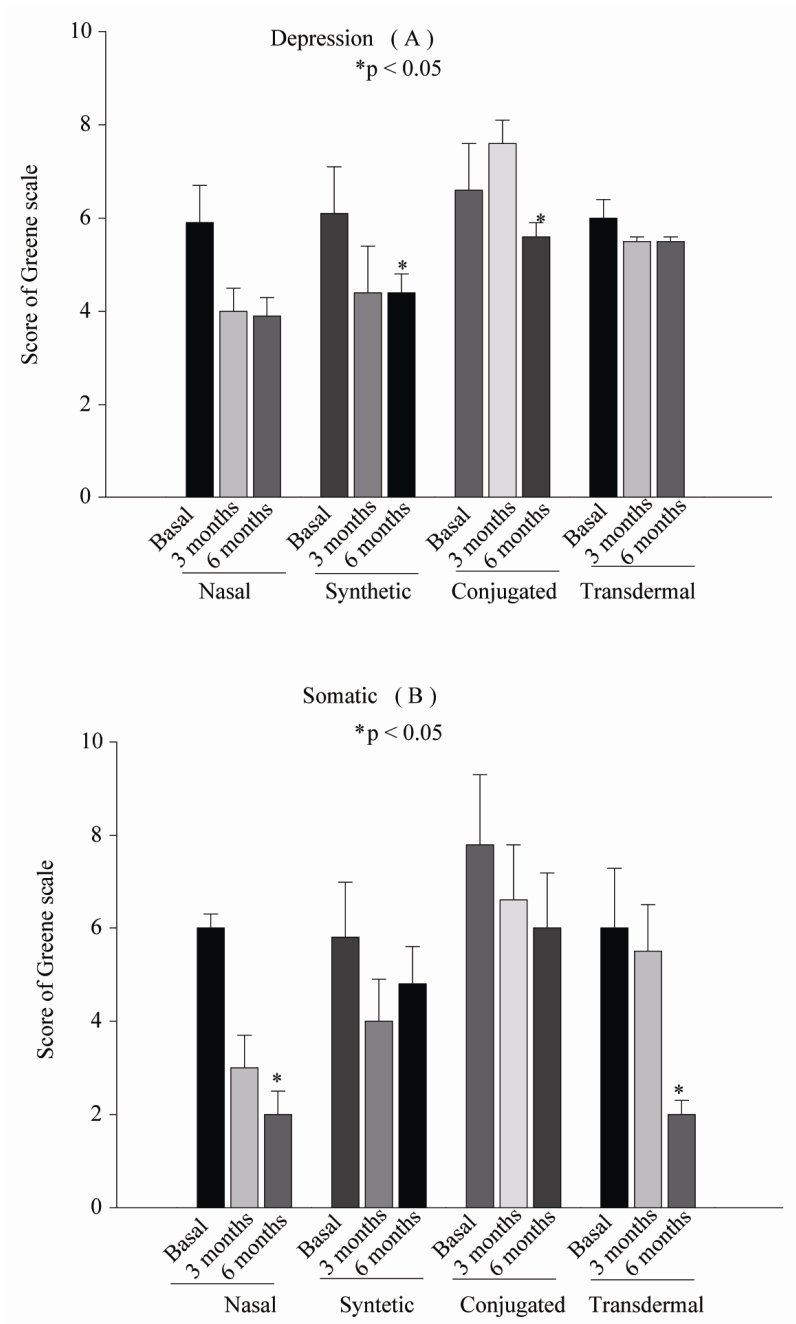

Figure 2. Significant changes in depression (Figure 2(A)) are shown with the use of synthetic steroids and conjugated estrogens. Somatic component (Figure 2(B)) demonstrated statistical difference with nasal spray and transdermal patches. In all types of therapy, a pattern of irregular changes was observed.

Significant changes in the psychological component were observed with the use of synthetic oral steroids and with transdermal patches. With the other types of HT there was a non-significant decrease (Figure 3(A)). The anxiety component showed a significant difference with the use of the nasal spray and with oral conjugated estrogens, although all forms of HT in this component showed an irregular pattern of changes (Figure 3(B)).

\section{DISCUSSION}

The present study in which the Greene scale score was used allowed us to evaluate the therapeutic commercial presentations of HT with the use of different pharmacological formulas — nasally, orally or transdermally_-for the treatment of postmenopausal symptoms. 

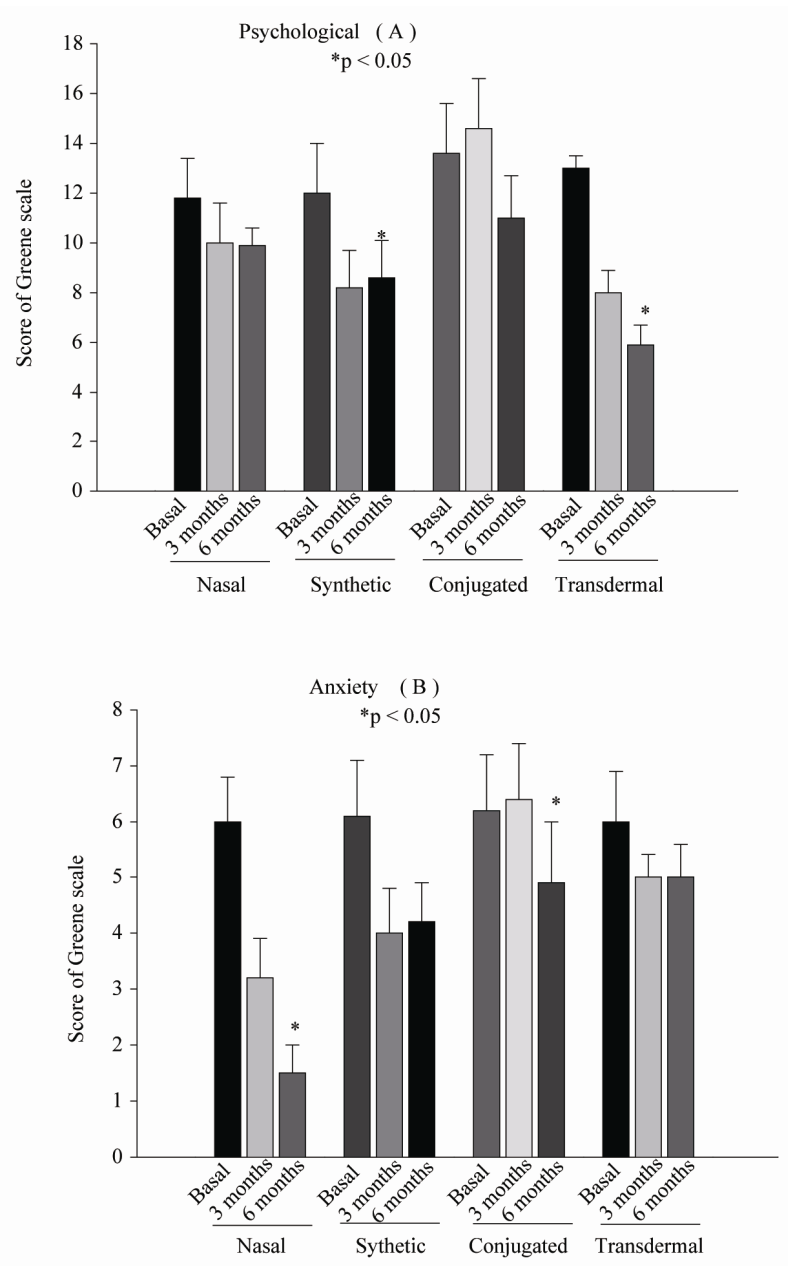

Figure 3. Psychological (Figure 3(A)) and anxiety (Figure 3(B)) components demonstrate changes with a tendency to decrease symptoms, but only with differences in some therapeutic forms as observed in the bar for each group.

We observed that the evaluated components show different responses in the control of postmenopausal symptoms.

We observed that the vasomotor and sexual component had a good response with all types of treatments used in this study. However, evaluation of the sexual component did not demonstrate improvement with regular pattern with the use of synthetic steroids. Depressive and somatic components had an irregular response with all the different therapeutic modalities. Because the profile of symptoms decrease was inconsistent, psychological and anxiety components also had a satisfactory response with only some of the therapeutic modalities. The difference was significant for up to 6 months of therapy. This demonstrates an equivalent efficacy on using a daily intranasal application of $300 \mu \mathrm{g} /$ day of $17 \beta$-estradiol, $0.625 \mathrm{mg}$ orally, $1.5 \mathrm{mg}$ transdermally and $2.5 \mathrm{mg}$ synthetic steroids to improve perimenopausal symptoms.
It should, however, be mentioned that each method has a different kinesis: the intranasal route has a pulse activity each day of application and the oral route has a sustained activity during the entire day of administration $[12,13]$. Changes in the response to each route of administration and medication used may be due to the fact that estrogens demonstrate their metabolic effects at the intracellular level because they interact with specific receptors in the cells of the effector organs to form dimerization that stimulates DNA synthesis. Therefore, estradiol is primarily metabolized in the liver where its principal metabolites are estriol and estrone, which are less active although also metabolized in the liver [14]. For its part, transdermal therapy delivers estrogen unaltered directly into the bloodstream, bypassing the liver, whereas tibolone is metabolized in three ways and according to the tissue acts as estrogen, androgen and progestagenic activity [15]. It should be kept in mind that absorption variability may exist among women and the different routes used; therefore, variability may also exist in the control of symptoms, which should be taken into consideration when selecting the appropriate route of administration in patients who will begin HT.

Scoring variation on the Greene scale has demonstrated great efficacy to quantify the incidence and intensity of perimenopausal symptoms, which have been widely validated. In no case was endometrial swelling observed. This was closely observed because estradiol was used as monotherapy for a 6-month period to exclusively evaluate HT response with differences in routes of administration. The Women's Health Initiative (WHI) study demonstrated that a relative risk exists with the use of estrogens associated with progestin [16]. There were also no cases of adverse effects such as mastodynia and transvaginal spotting. There were no clinical changes observed related to peripheral venous problems, which could suggest a state of hypercoagulation during the period of observation. One of the criteria for study inclusion was no vascular history or peripheral venous insufficiency.

All routes of administration demonstrated beneficial clinical effects for the control of postmenopausal symptoms. In order to achieve this, it is important to mention that patients with gynecological and schizoaffective disorders should significantly participate in the accomplishment of prophylactic indications and HT. Also, HT should be adapted according to the requirements of each patient and should be approached on the basis of personal history and expectations of HT. However, longterm use of estrogen may have potential risks, vaginally delivered estrogen therapies (cream, tablet or ring) are also very effective for treating vaginal dryness after menopause. But the $\mathrm{TH}$ have both protective benefits 
and potential risks like breast cancer, stroke or heart attack or heart attack and blood clot development, since older women, obese/diabetic women have much higher levels of a marker associated with inflammation, protein kinase C (PKC). Estrogen can either exacerbate or inhibit inflammatory processes depending upon whether PKC or protein kinase A (PKA) is constitutively active within cells. This fact probably explains the seemingly conflicting results of estrogen-only therapy between younger and older women; old age is associated with higher levels of inflammation markers like PKC. Furthermore, since estrogen also produces a biphasic response, younger, normal-weight women are best advised to take only physiological doses of the natural hormone.

We can conclude from these results that HT should be initiated for relief of symptoms through suitable selection of the route of administration and drug that has the greatest effect on the predominant symptoms of each patient, for which medical evaluation was sought.

\section{ACKNOWLEDGEMENTS}

The authors thank the personnel of the Endocrine Research Unit of the Specialties Hospital, National Medical Center of the Instituto Mexicano del Seguro Social for medical care and follow-up. Additionally, we thank Sharon Morey for editorial assistance in this manuscript.

The study was financed in part by the Fondo para la Investigaciión en Salud (FIS) of the IMSS and by the National Council Investigators (SNI).

\section{REFERENCES}

[1] Hansen, L.B. and Portman, D. (2006) Hormone therapy update. Current recommendations for menopausal symptoms. US Pharmacist, 31, 86-89.

[2] Smith, A.L. and Wein, A.J. (2010) Estrogen replacement therapy for the treatment of postmenopausal genitourinary tract dysfunction. Discovery Medicine, 10, 500-510.

[3] Dennerstein, L. and Lehert, P. (2004) Women's sexual functioning, lifestyle, mid-age, and menopause in 12 European countries. Menopause, 6, 778-785. doi:10.1097/01.GME.0000138544.50670.CC

[4] Reimer, A. and Johnson, L. (2011) Atrophic vaginitis: Signs, symptoms, and better outcomes. The Nurse Practitioner, 36, 22-28.

[5] Kingsberg, S., Kellogg, S. and Krychman, M. (2010) Treating dyspareunia caused by vaginal atrophy: A review of treatment options using vaginal estrogen therapy. International Journal of Women's Health, 9, 105-111.

[6] Writing Group for the Women's Health Initiative Investigators (2002) Risks and benefits of estrogen plus pro- gestin in healthy postmenopausal women: Principal results from the Women's Health Initiative randomized controlled trial. The Journal of the American Medical Association, 288, 321-333.

[7] Bond, S. and Horton, L.S. (2010) Management of postmenopausal vaginal symptoms in women. Journal of Gerontological Nursing, 36, 3-7. doi:10.3928/00989134-20100527-96

[8] Lacroix, A.Z., Chlebowski, R.T., Manson, J.E., Aragaki, A.K., Johnson, K.C., Martin, L., Margolis, K.I., Stefanick, M.I., Brzyski, R., Curb, J.D., Howard, B.V., Lewis, C.E. and Wactawski-Wende, J. (2011) Health outcomes after stopping conjugated equine estrogens among postmenopausal women with prior hysterectomy. The Journal of the American Medical Association, 30, 1305-1314. doi:10.1001/jama.2011.382

[9] Lowe, D.A., Baltgalvis, K.A. and Greising, S.M. (2010) Mechanisms behind estrogen's beneficial effect on muscle strength in females. Exercise and Sport Sciences Reviews, 38, 61-67. doi:10.1097/JES.0b013e3181d496bc

[10] Basurto, L., Saucedo, R., Zarate, A., Martinez, C., Gamino, C.E. and Reyes, E. (2006) Hernandez-Valencia M. Effect of pulsed estrogen therapy on hemostatic markers in comparison with oral estrogens regimen in postmenopausal women. Ginecologlay and Obstetricia Investigation, 61, 61-64. doi:10.1159/000088603

[11] Hernández-Valencia, M., Córdova-Pérez, N., Basurto, L., Saucedo, R., Vargas, C., Vargas, A., Ruiz, M., Manuel, L. and Zárate, A. (2010) Frequency of symptoms of the climacteric syndrome. Ginecologlay Obstetricia de Mexico, 78, 232-237.

[12] Henderson, V.W. (2010) Action of estrogens in the aging brain: dementia and cognitive aging. Biochimica et Biophysica Acta, 1800, 1077-1083.

[13] Rachon, D., Suchecka-Rachon, K., Hak, L. and Mysliwska, J. (2006) Effects of intranasal 17- $\beta$ estradiol administration on serum bioactive interleukin-6 and C-reactive protein levels in healthy postmenopausal women. Menopause, 5, 840-845.

[14] Gompel, A. (2006) Molecular action of estrogens and anti-estrogens. Maturitas, 54, 313-314. doi:10.1016/j.maturitas.2006.04.015

[15] Simoncini, T., Mannella, P., Fornari, L., Caruso, A., Varone, G., Garibaldi, S. and Genazzani, A. (2004) Tibolone activates nitric oxide synthesis in human endothelial cells. Journal of Clinical Endocrinology \& Metabolism, 89, 4594-4600. doi:10.1210/jc.2003-032189

[16] The Women's Health Initiative Steering Committee. (2004) Effects of conjugated equine estrogens in postmenopausal women with hysterectomy: The Women's Health Initiative randomized, controlled trial. The Journal of the American Medical Association, 291, 17011712. doi:10.1001/jama.291.14.1701 